\title{
Efecto de la sustitución de zinc por níquel en sólidos del tipo hidrotalcita
}

\section{Effect of zinc substitution by nickel on solids with hydrotalcite type structure}

\author{
Johana Rodríguez-Ruiz ${ }^{1}$, Shirley Osorio-Herrera ${ }^{1}$, \\ Edgardo Meza-Fuentes ${ }^{2}$
}

\author{
${ }^{1}$ Grupo de Investigación en Procesos de la Industria Petroquímica, SENA-Centro para la Industria Petroquímica, Carta- \\ gena, Colombia. \\ e-mail: ji.rodriguez@misena.edu.co,sosorioh@sena.edu.co \\ ${ }^{2}$ Grupo de Estudios en Materiales y Combustibles, Universidad de Cartagena, Campus San Pablo, Cartagena, Colombia \\ e-mail: emezaf@unicartagena.edu.co
}

\section{RESUMEN}

Las hidrotalcitas pueden ser usadas para diferentes aplicaciones debido a sus propiedades químicas y por dar origen a sólidos con elevada área superficial específica, buena estabilidad térmica, alta dispersión y carácter básico. Estas características han conducido a que estos materiales hayan recibido mucha atención y aplicación en áreas como la remoción de contaminantes y en la síntesis de catalizadores. En este estudio se evaluó el efecto de la sustitución de zinc por níquel en hidrotalcitas a base de zinc-aluminio, las cuales han mostrado actividad para la depolimerización catalítica de poli (tereftalato de etileno). La estructura del tipo hidrotalcita fue observada en todos los materiales, notándose que el aumento de zinc conduce a mayores valores del parámetro $a$ de las celdas ortorrómbicas de las hidrotalcitas. La disminución del parámetro $c$ condujo a una mayor interacción $\mathrm{CO}_{3}{ }^{2-} \cdot \cdots \mathrm{OH}$ en la región interlaminar. Los sólidos con mayor contenido de zinc colapsaron a menores temperaturas, efecto contrario al inducido por el níquel. Los sólidos calcinados contienen las fases $\mathrm{ZnO}, \mathrm{NiO}$ y $\gamma-\mathrm{Al}_{2} \mathrm{O}_{3}$, cuya presencia depende de los metales presentes. Los resultados sugieren la presencia de partículas grandes y con alta cristalinidad de la fase $\mathrm{ZnO}$, mientras que los picos definidos y anchos de $\mathrm{NiO}$ sugieren la existencia de partículas pequeñas de esta especie, cuya cristalinidad está afectada por la presencia de cationes $\mathrm{Zn}^{2+}$ y $\mathrm{Al}^{3+}$ en la red cristalina cubica del NiO. Los materiales conteniendo dos metales presentaron isotermas características de materiales predominantemente macroporosos, En relación a los materiales con tres metales, las estructuras son mesoporosas y con alta área superficial específica, observándose que el sólido $\mathrm{Zn}_{0,44} \mathrm{Ni}_{0,22} \mathrm{Al}_{0,33}$ contiene poros en forma de hendidura, mientras que los sólidos $\mathrm{Zn}_{0,33} \mathrm{Ni}_{0,33} \mathrm{Al}_{0,33}$ y $\mathrm{Ni}_{0,44} \mathrm{Zn}_{0,22} \mathrm{Al}_{0,33}$ presentaron isotermas propias de materiales con poros estrechos en forma de capilares.

Palabras clave: Hidrotalcita; Zinc, Colapso de Estructura; Formación de Óxidos.

\begin{abstract}
Hydrotalcites can be used for different applications due to their chemical properties and to give rise to solids with high specific surface area, good thermal stability, high dispersion and basic character. These characteristics have led to the fact that these materials have received much attention and application in areas such as the removal of contaminants and the synthesis of catalysts. This study evaluated the effect of zinc substitution by nickel on zinc-aluminum hydrotalcites, which have shown activity for the catalytic depolymerization of poly(ethylene terephthalate). The hydrotalcite type structure was observed in all materials, noting that the increase of zinc leads to higher values of a parameter of the orthorhombic cells of the hydrotalcites. The decrease of the $\mathrm{c}$ parameter led to a greater interaction $\mathrm{CO}_{3}{ }^{2-} \cdot \cdot \mathrm{OH}$ in the interlaminar space. Solids with higher zinc content collapsed at lower temperatures, contrary to that induced by nickel. The calcined solids contain the $\mathrm{ZnO}, \mathrm{NiO}$ y $\gamma-\mathrm{Al}_{2} \mathrm{O}_{3}$ phases, whose presence depends on the metals present. The results suggest the presence of large particles with high crystallinity of the $\mathrm{ZnO}$ phase, whereas the broad peaks of NiO suggest the existence of small particles of this phase, whose crystallinity is affected by the presence of $\mathrm{Zn}^{2+}$ and $\mathrm{Al}^{3+}$ in the cubic lattice of $\mathrm{NiO}$. Materials containing two metals presented isotherms characteristic of predominantly macroporous solids. In relation to the materials with three metals, the structures are mesoporous
\end{abstract}


and with high specific surface area, observing that the solid $\mathrm{Zn}_{0,44} \mathrm{Ni}_{0,22} \mathrm{Al}_{0,33}$ contains slit-shaped pores, while the solids $\mathrm{Zn}_{0,33} \mathrm{Ni}_{0,33} \mathrm{Al}_{0,33}$ and $\mathrm{Ni}_{0,44} \mathrm{Zn}_{0,22} \mathrm{Al}_{0,33}$ presented typical isotherms of materials with narrow pores in the form of capillaries.

Keywords: Hydrotalcite; Zinc; Structure Collapse; Oxides Formation.

\section{INTRODUCCIÓN}

Las hidrotalcitas son materiales que debido a sus propiedades químicas pueden ser usadas para diferentes aplicaciones o para dar origen a través de procesos térmicos una gran variedad de materiales, entre los que se destacan óxidos mixtos o mezclas de óxidos que poseen propiedades útiles para diferentes propósitos, tales como: elevada área superficial específica, buena estabilidad térmica y mecánica, alta dispersión de partículas y un carácter básico o acido que depende de los cationes e iones presentes en la estructura original. Estas características han conducido a que estos materiales hayan recibido mucha atención y aplicación en aspectos como la remoción de contaminantes, síntesis de catalizadores, estabilizantes de polímeros, medicina, entre otros [1-3].

Los sólidos con estructura del tipo hidrotalcita, también llamados hidróxidos dobles laminares, son representados por la fórmula general $\left[\mathrm{M}^{2+}{ }_{1-x} \mathrm{M}^{3+}{ }_{x}(\mathrm{OH})_{2}\right]\left(\mathrm{A}^{\mathrm{n}-}\right)_{x / \mathrm{n}} \cdot \mathrm{mH}_{2} \mathrm{O}$, en donde $\mathrm{M}^{2+}$ es uno o varios catiónes divalentes $\left(\mathrm{Mg}^{2+} \mathrm{Zn}^{2+}, \mathrm{Ni}^{2+}\right.$, etc.) $\mathrm{M}^{3+}$ es uno o varios cationes trivalentes $\left(\mathrm{Al}^{3+}, \mathrm{Fe}^{3+}, \mathrm{Cr}^{3+}\right.$, etc. $)$; $\mathrm{A}^{\mathrm{n}-}$ es un anión compensador de la carga y $x$ es definido como la relación $\mathrm{M}^{3+} / \mathrm{M}^{2+}+\mathrm{M}^{3+}$ [1]. Las hidrotalcitas están constituidas por celdas unitarias ortorrómbicas con una simetría $R-3 m$, con el valor del parámetro $c$ siendo tres veces la distancia entre dos laminas sucesivas. El parámetro de celda $c$ es determinado por el tamaño y por la cantidad de aniones presentes entre las láminas, mientras que el parámetro de celda $a$, depende del tipo y de la cantidad de los cationes presentes [2]. En relación a los aniones, el más estudiado es el carbonato debido a la estabilidad del mismo y a que este por efecto de la temperatura se descompone a dióxido de carbono. Otros aniones empleados en la síntesis de hidrotalcitas son; $\mathrm{F}^{-}, \mathrm{Cl}^{-}, \mathrm{Br}^{-}, \mathrm{I}^{-},\left(\mathrm{ClO}_{4}\right)^{-},\left(\mathrm{NO}_{3}\right)^{-},\left(\mathrm{ClO}_{3}\right)^{-}$, $\left(\mathrm{IO}_{3}\right)^{-}, \mathrm{OH}^{-},\left(\mathrm{CrO}_{4}\right)^{2-}$ así como polioxometalatos y aniones orgánicos [4-8].

Es de destacar que, debido a sus propiedades de adsorción las hidrotalcitas pueden ser usadas para remover diversos oxoaniones, aniones inorgánicos [9], y otras especies como uranio en aguas residuales; lo cual está jugando un papel central en la industria nuclear, ya que de esta forma se disminuye su toxicidad radiológica y biológica [10].

En relación al campo de la catálisis, las hidrotalcitas se pueden usar para sintetizar óxidos metálicos mixtos, con buenas áreas superficiales y con un carácter básico que las hace útiles en un número elevado de reacciones. En este contexto se han realizado diversos estudios, lográndose evidenciar la actividad de sólidos derivados de hidrotalcitas en reacciones como; la transesterificación de aceite de soja con metanol para producir biodiesel usando catalizadores a base de Zn-Al-Zr (zinc, aluminio y zirconio) [11]; reacción de desplazamiento del vapor de agua (WGS, wáter gas shift) con catalizadores de óxido de níquel, zinc y aluminio, provenientes de un precursor del tipo hidrotalcita [12,13]; deshidrogenación oxidativa de propano (DHOP) usando catalizadores obtenidos de precursores del tipo hidrotalcita a base de NiZnFe y NiMgFe modificados con especies de $\mathrm{V}$ y Cr (vanadio y cromo) [14]; esterificación de glicerol usando catalizadores con carácter básico como óxidos mixtos de Mg-Fe (magnesio y hierro) derivados de hidrotalcitas [15]; depolimerización de poli (tereftalato de etileno) vía glicolisis con etilenglicol usando óxidos mixtos de magnesio y aluminio proveniente de hidrotalcitas [16], entre otras.

En este estudio se evaluó el efecto de la sustitución de zinc por níquel en hidrotalcitas a base de zincaluminio, las cuales han mostrado por estudios previamente realizados en nuestros grupos de investigación, actividad para la depolimerización catalítica de poli (tereftalato de etileno). De igual manera se analizó el efecto de la sustitución en los sólidos obtenidos por procesos de calcinación a $500{ }^{\circ} \mathrm{C}$.

\section{MATERIALES Y MÉTODOS}

\subsection{Síntesis de Materiales}

Se prepararon dos tipos de precursores del tipo hidrotalcita, el primero conteniendo dos elementos metálicos ( $\mathrm{Zn}-\mathrm{Al}$ y Ni-Al) y el segundo conteniendo tres elementos metálicos ( $\mathrm{Zn}-\mathrm{Ni}-\mathrm{Al})$. Para el primer tipo de solidos se preparó una solución salina conteniendo nitrato de zinc $\left[\mathrm{Zn}\left(\mathrm{NO}_{3}\right)_{2} \bullet 6 \mathrm{H}_{2} \mathrm{O}\right]$ o de níquel $\left[\mathrm{Ni}\left(\mathrm{NO}_{3}\right)_{2} \bullet 6 \mathrm{H}_{2} \mathrm{O}\right]$ y nitrato de aluminio $\left[\mathrm{Al}\left(\mathrm{NO}_{3}\right)_{3} \cdot 9 \mathrm{H}_{2} \mathrm{O}\right]$, la cual se mezcló bajo agitación constante con una segunda solución conteniendo hidróxido de potasio $(\mathrm{KOH})$ y carbonato de potasio $\left(\mathrm{K}_{2} \mathrm{CO}_{3}\right)$, manteniéndose el pH en 8.3. El sólido formado se filtró y se lavó con agua desionizada a $60{ }^{\circ} \mathrm{C}$ hasta la eliminación total de la especie nitra- 
to. El material resultante se secó a $60{ }^{\circ} \mathrm{C}$ durante 24 h y seguidamente se calcinó en atmosfera de aire a 500 ${ }^{\circ} \mathrm{C}$ por $4 \mathrm{~h}$.

Para el caso de la síntesis de los precursores conteniendo los tres elementos metálicos se procedió de forma similar a la descripción anterior, con la diferencia de haber preparado la solución salina inicial con los nitratos de zinc, níquel y aluminio. En relación al proceso de lavado, secado y calcinación, se usaron las mismas condiciones empleadas durante la síntesis de las hidrotalcitas bimetálicas.

Las muestras obtenidas se codificaron de acuerdo al número de moles de los metales presentes en los materiales, originando los sólidos bimetálicos $\mathrm{Zn}_{0,66} \mathrm{Al}_{0,33}$ y $\mathrm{Ni}_{0,66} \mathrm{Al}_{0,33}$, y los sólidos trimetálicos $\mathrm{Zn}_{0,44} \mathrm{Ni}_{0,22} \mathrm{Al}_{0,33}, \mathrm{Zn}_{0,33} \mathrm{Ni}_{0,33} \mathrm{Al}_{0,33}$ y $\mathrm{Ni}_{0,44} \mathrm{Zn}_{0,22} \mathrm{Al}_{0,33}$.

\subsection{Caracterización de los Materiales}

Los sólidos sintetizados fueron caracterizados por las técnicas de fluorescencia de rayos X (XRF, Thermo Fisher, Optiquant Analysis), espectrometría en la región del infrarrojo con transformada de Fourier (FTIR, Shimadzu, modelo IRAffinity), análisis termogravimétrico (TGA, TA Instruments modelo SDT Q600) y analisis diferencial de la masa con respecto a la temperatura (DTG, TA Instruments modelo SDT Q600), difracción de rayos X (DRX, Xpert PAanalytical Empyrean Serie II, Modelo 2012), medida del área superficial específica y porosimetría por adsorción de nitrógeno a $77 \mathrm{~K}$ (Micromeritics, ASAP 2020).

Para la caracterización de las hidrotalcitas por fluorescencia de rayos X las muestras fueron previamente molidas y tamizadas a 200 mesh para garantizar la homogeneidad de las mismas. Posteriormente fueron analizadas usando las bandas de emisión $\mathrm{K} \alpha$ a $1.48 \mathrm{keV}$ para aluminio, $\mathrm{K} \alpha$ a $7.97 \mathrm{keV}$ para níquel y $\mathrm{K} \alpha$ a $9.57 \mathrm{keV}$ para zinc.

Para los análisis por espectroscopia infrarroja, las muestras se mezclaron en una relación de 1/100 en masa con bromuro de potasio, triturándose la mezcla hasta obtener un polvo fino que fue posteriormente prensado para obtener una pastilla que fue analizada en el equipo. Los espectros se registraron en la región del infrarrojo medio comprendida entre 4000 y $400 \mathrm{~cm}^{-1}$, usando 32 barridos y una resolución de $4 \mathrm{~cm}^{-1}$.

Para los análisis termogravimétricos (TGA) y diferencial de la masa con respecto a la temperatura (DTG) de los precursores del tipo hidrotalcita se usaron de 3 a $5 \mathrm{mg}$ de muestra, los cuales fueron sometidos a una velocidad de calentamiento de $10{ }^{\circ} \mathrm{C} / \mathrm{min}$, desde $30{ }^{\circ} \mathrm{C}$ hasta la temperatura en la cual dejo de registrarse pérdida de masa, usando un flujo de $50 \mathrm{~mL} / \mathrm{min}$ de aire.

Los difractogramas de rayos $\mathrm{X}$ de las hidrotalcitas fueron obtenidos usando radiación $\mathrm{Cu} \mathrm{K} \alpha$, generada a $40 \mathrm{kV}$ y $30 \mathrm{~mA}$. Los análisis se realizaron en el intervalo de 10 a $65\left(2 \theta^{\circ}\right)$, y un paso de $0,03 \%$. Para el caso de los sólidos calcinados el intervalo de análisis fue de 10 a $80\left(2 \theta^{\circ}\right)$, y un paso de $0,03^{\circ} / \mathrm{s}$.

El área superficial y la porosidad de los sólidos calcinados fueron medidos por medio de la adsorcióndesorción de nitrógeno a $-196.15{ }^{\circ} \mathrm{C}$ usando el método BET (Brunauer-Emmett-Teller) para el caso del área superficial específica [17], y el método BJH (Barrett-Joyner-Halenda) para el caso de la porosidad (diámetro de poro) [18]. Las isotermas de adsorción-desorción fueron obtenidas a través del volumen de nitrógeno adsorbido a la temperatura del nitrógeno líquido en diferentes valores de $\mathrm{p} / \mathrm{p}^{\circ}$. Antes de proceder con el análisis, los sólidos fueron calentados a $200{ }^{\circ} \mathrm{C}$ y sometidos a una presión de vacío de $2 \mu \mathrm{mHg}$, con el fin de remover el agua y el material volátil presente en los sólidos.

\section{RESULTADOS Y DISCUSIÓN}

Los resultados de XRF mostraron que las relaciones molares de las soluciones usadas durante la síntesis se mantuvieron en los sólidos obtenidos, lo cual confirmó que el método de preparación fue el adecuado para la obtención de las hidrotalcitas. En concordancia con la cuantificación elemental, todos los materiales presentaron difractogramas característicos de la fase del tipo hidrotalcita, siendo observado que el material con mayor contenido de zinc $\left(\mathrm{Zn}_{0,66} \mathrm{Al}_{0,33}\right)$ mostro los picos más agudos y simétricos, lo cual sugiere el mayor grado de cristalinidad de este material.

Las principales diferencias en los difractogramas se observaron en la posición de los picos correspondientes a los planos $\left(\begin{array}{lll}O & O & l\end{array}\right)$ y $(h k 0)$, los cuales están relacionados con los parámetros de celda $c$ y $a$ de la estructura ortorrómbica de las hidrotalcitas. Este efecto se evidenció a través de los picos de los planos $\left(\begin{array}{llll}0 & 0 & 3\end{array}\right)$ y ( 1110$)$, mostrados en la Figura 1, en los que se observaron variaciones en la simetría y posición de los picos, originadas por las diferencias en los parámetros de celda entre los sólidos, cuyos valores son reportados en la Tabla 1. Adicionalmente se observó que el aumento del contenido de zinc condujo al aumento del tamaño de las láminas en la estructura del tipo hidrotalcita, el cual está directamente relacionado con el parámetro $a$ y con el tamaño de los cationes presentes, siendo registrado que el zinc $\left(\mathrm{Zn}^{2+} ; 0,74 \AA\right.$ ) posee mayor radio iónico que el níquel $\left(\mathrm{Ni}^{2+} ; 0,69 \AA\right)[1]$. 
Todos los materiales presentaron en la región del infrarrojo medio, espectros característicos de los compuestos del tipo hidrotalcita, en los cuales se evidenciaron bandas asociadas a grupos hidroxilo $\left(\mathrm{OH}^{-}\right)$ presentes en las láminas, en torno a los $3400 \mathrm{~cm}^{-1}$, así como bandas asociadas a aniones carbonato $\left(\mathrm{CO}_{3}{ }^{2-}\right)$ a $1383 \mathrm{~cm}^{-1}$ y a moléculas de agua a través de la banda a $1640 \mathrm{~cm}^{-1}$, como se observa en la Figura 2 [19-21]. En el caso de los solidos $\mathrm{Zn}_{0,66} \mathrm{Al}_{0,33}$ y $\mathrm{Zn}_{0,44} \mathrm{Ni}_{0,22} \mathrm{Al}_{0,33}$ se evidencio un hombro cercano a los $1525 \mathrm{~cm}^{-1}$ que puede estar asociado a interacciones de los aniones carbonato y grupos hidróxido $\left(\mathrm{CO}_{3}{ }^{2-} \cdot \cdots \mathrm{OH}\right)$ originadas por la menor distancia interlaminar registrada en estos materiales, la cual está directamente relacionada con los valores del parámetro c, mostrados en la Tabla 1 [22,23].

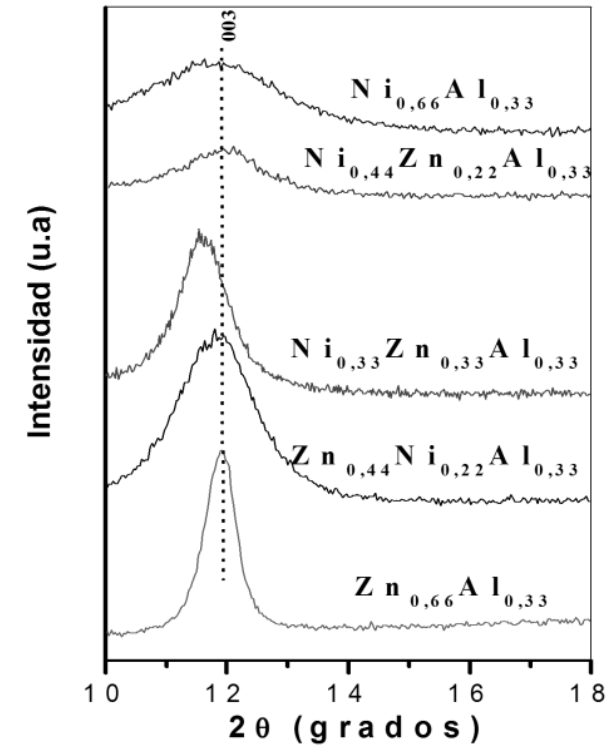

a

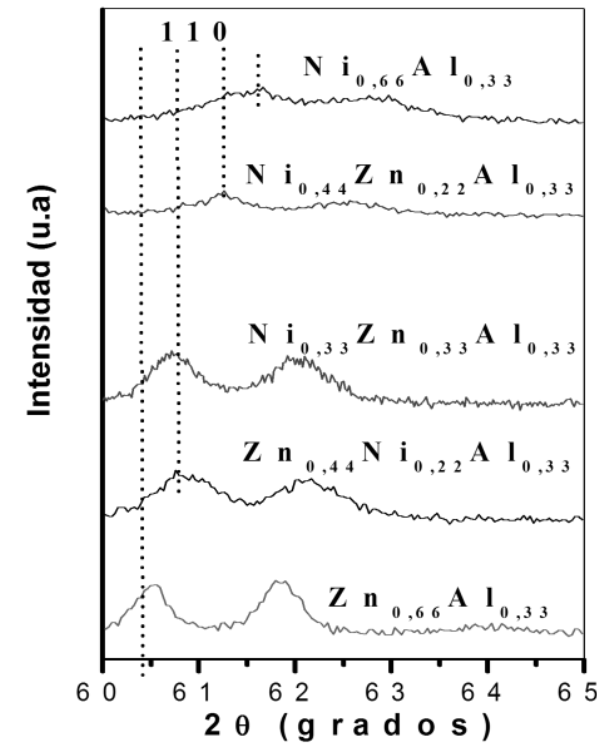

b

Figura 1: Difractogramas de rayos $\mathrm{X}$ en los que se muestran los picos correspondientes a los planos $\left(\begin{array}{llll}0 & 0 & 3\end{array}\right)$ y $\left(\begin{array}{lll}1 & 1 & 0\end{array}\right)$, característicos de los parámetros $c$ (1a) y $a(1 \mathrm{~b})$, respectivamente.

Tabla 1: Parámetros de celda $c$ y a calculados a través de los picos correspondientes a los planos $\left(\begin{array}{llll}0 & 0 & 3\end{array}\right)$ y $\left(\begin{array}{lll}1 & 1 & 0\end{array}\right)$.

\begin{tabular}{ccc}
\hline MUESTRA & PARÁMETRO $\boldsymbol{c}$ & PARÁMETRO $\boldsymbol{a}$ \\
\hline $\mathrm{Zn}_{0,66} \mathrm{Al}_{0,33}$ & 7,437 & 3,058 \\
$\mathrm{Ni}_{0,66} \mathrm{Al}_{0,33}$ & 7,519 & 3,012 \\
$\mathrm{Zn}_{0,44} \mathrm{Ni}_{0,22} \mathrm{Al}_{0,33}$ & 7,437 & 3,041 \\
$\mathrm{Zn}_{0,33} \mathrm{Ni}_{0,33} \mathrm{Al}_{0,33}$ & 7,627 & 3,041 \\
$\mathrm{Ni}_{0,44} \mathrm{Zn}_{0,22} \mathrm{Al}_{0,33}$ & 7,459 & 3,024 \\
\hline
\end{tabular}




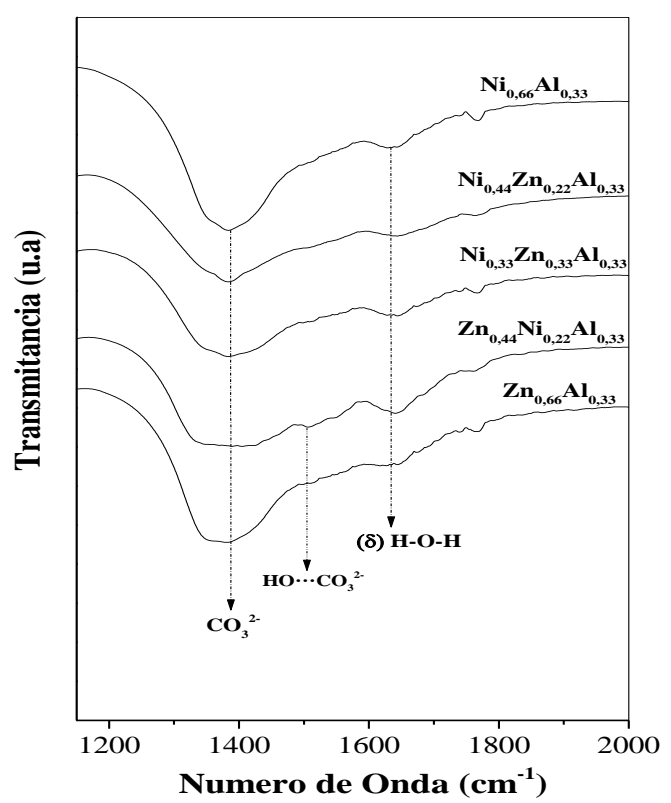

Figura 2: Espectros en la región del infrarrojo en el intervalo de $1180-2000 \mathrm{~cm}^{-1}$ de los sólidos del tipo hidrotalcita.

Las curvas de termogravimetría (TGA) y diferencial de masa con respecto a la temperatura $(\mathrm{dm} / \mathrm{dt})$ son mostradas en las Figuras 3-4. En todos los casos se evidenciaron zonas de pérdida de masa asociadas a la salida del agua fisisorbida y del agua localizada en las regiones interlaminares, seguidas del colapso de la estructura del tipo hidrotalcita por el inicio de la descomposición de grupos hidróxido y de los aniones carbonato, lo que finalmente condujo a la formación de los óxidos de los metales presentes. El colapso de la estructura se evidenció por una disminución abrupta en la velocidad de pérdida de masa a medida que aumenta la temperatura, cuyos valores son mostrados en la Tabla 2. Este efecto se observó claramente en todos los materiales, excepto el solido $\mathrm{Zn}_{0,66} \mathrm{Al}_{0,33}$, en el cual este fenómeno ocurrió a aproximadamente $173{ }^{\circ} \mathrm{C}$. Los sólidos con mayor contenido de zinc fueron los que colapsaron a menores temperaturas, mientras que el material conteniendo apenas níquel y aluminio fue el que colapso a mayor temperatura. En relación a la pérdida de masa al momento del colapso se observa a través de la tabla 2, que los solidos $\mathrm{Zn}_{0,44} \mathrm{Ni}_{0,22} \mathrm{Al}_{0,33}$ y $\mathrm{Ni}_{0,44} \mathrm{Zn}_{0,22} \mathrm{Al}_{0,33}$ fueron los que perdieron mayor cantidad de masa, lo cual se asocia a la mayor pendiente ocasionada por la salida del agua fisisorbida e interlaminar.
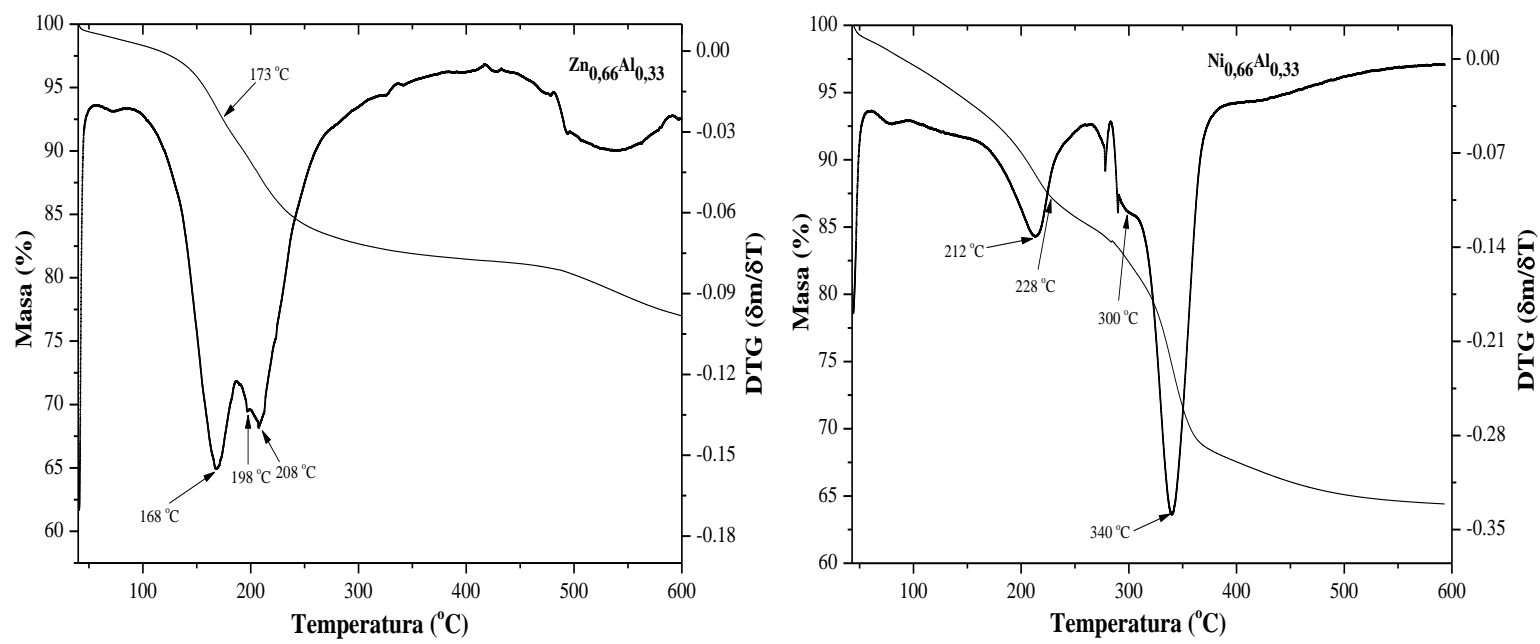

Figura 3: Curvas de termogravimetria (TGA) y diferencial de masa con respecto a la temperatura (dm/dt) de los solidos $\mathrm{Zn}_{0,66} \mathrm{Al}_{0,33}$ y $\mathrm{Ni}_{0,66} \mathrm{Al}_{0,33}$. 

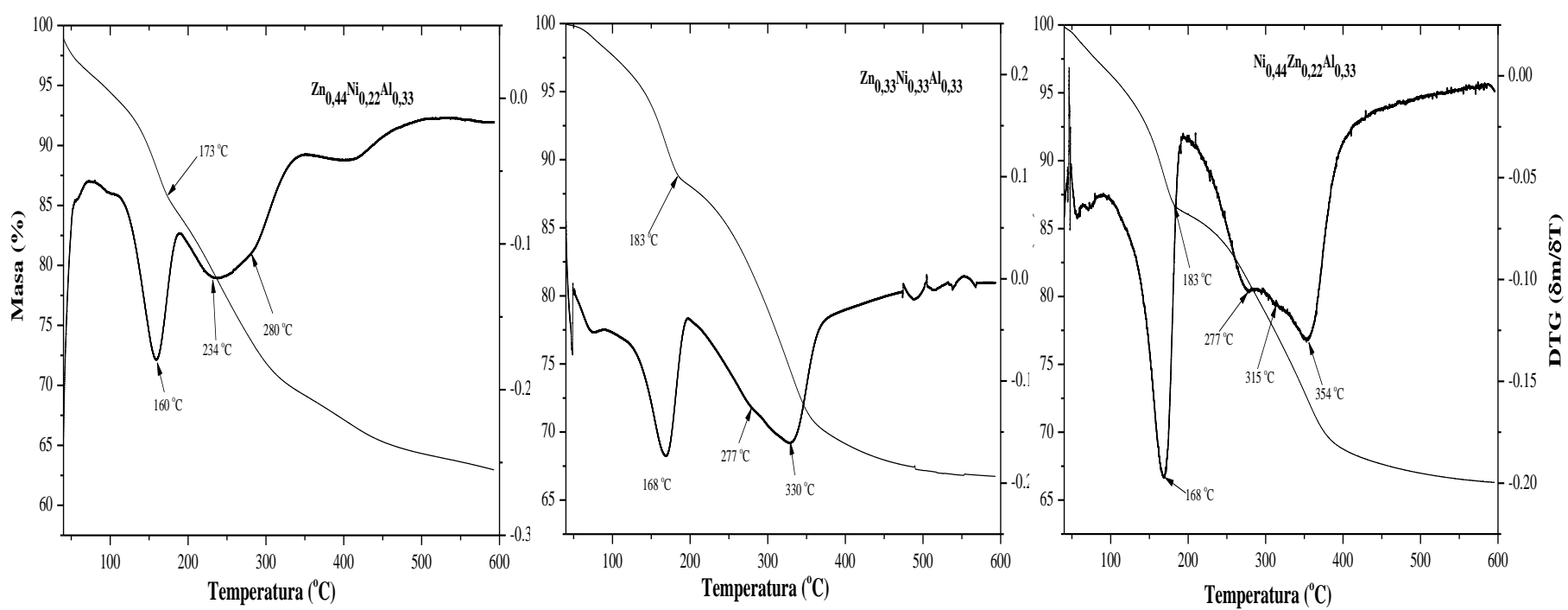

Figura 4: Curvas de termogravimetria (TGA) y diferencial de masa con respecto a la temperatura (dm/dt) de los solidos $\mathrm{Zn}_{0,44} \mathrm{Ni}_{0,22} \mathrm{Al}_{0,3}, 3, \mathrm{Zn0}{ }_{, 33} \mathrm{Ni}_{0,33} \mathrm{Al}_{0,33}$ y Ni $\mathrm{Ni}_{0,44} \mathrm{Zn}_{0,22} \mathrm{Al}_{0,33}$.

Tabla 2: Temperatura y pérdida de masa asociadas al colapso de la estructura del tipo hidrotalcita de los materiales.

\begin{tabular}{ccc}
\hline MUESTRA & TEMPERATURA DE COLAPSO $\left({ }^{\circ} \mathbf{C}\right)$ & PÉRDIDA DE MASA HASTA EL COLAPSO $(\%)$ \\
\hline $\mathrm{Zn}_{0,66} \mathrm{Al}_{0,33}$ & 173 & 7,35 \\
$\mathrm{Ni}_{0,66} \mathrm{Al}_{0,33}$ & 228 & 13,01 \\
$\mathrm{Zn}_{0,44} \mathrm{Ni}_{0,22} \mathrm{Al}_{0,33}$ & 173 & 14,30 \\
$\mathrm{Zn}_{0,33} \mathrm{Ni}_{0,33} \mathrm{Al}_{0,33}$ & 183 & 11,36 \\
$\mathrm{Ni}_{0,44} \mathrm{Zn}_{0,22} \mathrm{Al}_{0,33}$ & 183 & 13,36 \\
\hline
\end{tabular}

Después del colapso de la estructura continuó la pérdida de masa debido a la descomposición de los grupos hidróxido y carbonato, lo que condujo a la formación de los óxidos de zinc $(\mathrm{ZnO})$ y níquel $(\mathrm{NiO})$ [13]. Para el caso de los materiales con mayor contenido de zinc se observó a través de la curva DTG un pico a temperaturas inferiores a $300{ }^{\circ} \mathrm{C}$, asociado a la formación del $\mathrm{ZnO}$, el cual varía dependiendo de la composición de los sólidos. Este hecho puede deberse a que la presencia de níquel y de aluminio dificulta el acercamiento de las especies $\mathrm{Zn}^{2+}$ y consecuentemente la formación y crecimiento de las primeras partículas del $\mathrm{ZnO}$. Este efecto también ocurre para el caso del $\mathrm{NiO}$, el cual se forma a partir de $300{ }^{\circ} \mathrm{C}$, siendo observados picos entre 300 y $400{ }^{\circ} \mathrm{C}$, los cuales se deben a la formación de partículas del óxido a través de diferentes procesos de difusión de las especies $\mathrm{Ni}^{2+}$ en el volumen de los materiales, los cuales pueden ser favorecidos $\mathrm{u}$ obstaculizados por la presencia del zinc y del aluminio en los sólidos iniciales [12-13].

La pérdida de masa posterior a la formación de $\mathrm{ZnO}$ y $\mathrm{NiO}$ está asociada a la formación de óxidos de transición de aluminio o formación de aluminatos, así como a la descomposición de carbonatos que quedaron ocluidos en el interior de los materiales, producto del colapso de la estructura.

Los cambios en la masa relacionados con la formación de los óxidos de los metales presentes en las hidrotalcitas se confirmaron a través de los difractogramas de rayos $\mathrm{X}$ de los materiales calcinados a $500{ }^{\circ} \mathrm{C}$, como se observa en la Figura 5. Todos los sólidos presentaron difractogramas con picos característicos de $\mathrm{ZnO}, \mathrm{NiO}$ y $\gamma-\mathrm{Al}_{2} \mathrm{O}_{3}$, cuya presencia depende de los metales presentes. En algunos casos se evidencio la presencia de la fase boehmita, la cual es un oxihidroxido de aluminio que actúa como un precursor de óxidos de transición de aluminio. La presencia de picos bien definidos y agudos de $\mathrm{ZnO}$ indica la presencia de partículas grandes y con alta cristalinidad de esta fase, mientras que los picos definidos y anchos de $\mathrm{NiO}$ sugieren la existencia de partículas pequeñas de esta especie, las cuales también pueden tener alteraciones en la cristalinidad provocadas posiblemente por la presencia de cationes $\mathrm{Zn}^{2+}$ y $\mathrm{Al}^{3+}$ en la red cristalina cubica del $\mathrm{NiO}$ $[13,23]$. 


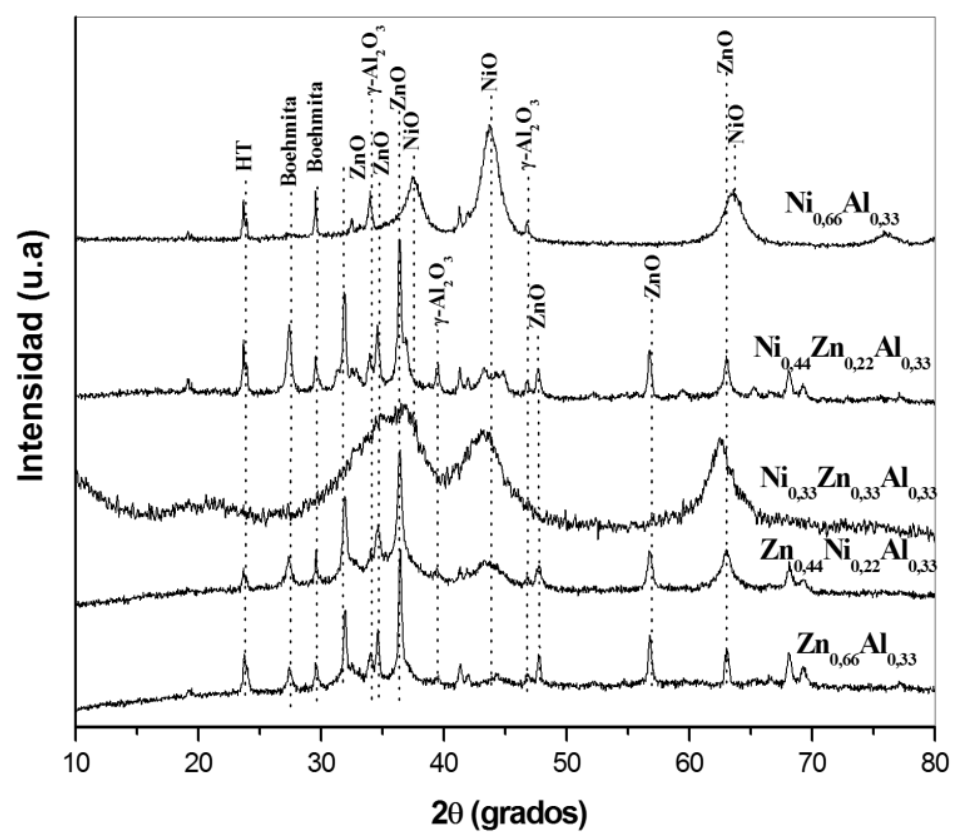

Figura 5: Difractogramas de rayos $X$ en el intervalo de 10-80 $2 \theta$ de los sólidos obtenidos al calcinar las hidrotalcitas a $500{ }^{\circ} \mathrm{C}$.

La Tabla 3 muestra los valores de los parámetros texturales de área superficial específica y diámetro de poros de los sólidos calcinados a $500{ }^{\circ} \mathrm{C}$. Se observa que el material conteniendo zinc y aluminio fue el que presento menor área superficial específica $\left(10 \mathrm{~m}^{2} / \mathrm{g}\right)$, mientras que los otros materiales poseen áreas cercanas a $70 \mathrm{~m}^{2} / \mathrm{g}\left(\mathrm{Ni}_{0,66} \mathrm{Al}_{0,33}\right.$ y $\left.\mathrm{Zn}_{0,44} \mathrm{Ni}_{0,22} \mathrm{Al}_{0,33}\right)$ y en torno a $180 \mathrm{~m}^{2} / \mathrm{g}\left(\mathrm{Zn}_{0,33} \mathrm{Ni}_{0,33} \mathrm{Al}_{0,33}\right.$ y $\left.\mathrm{Ni}_{0,44} \mathrm{Zn}_{0,22} \mathrm{Al}_{0,33}\right)$. El material $\mathrm{Zn}_{0,66} \mathrm{Al}_{0,33}$ originó una isoterma de adsorción-desorción (Figura 6) con ausencia de un punto de inflexión a bajos valores de $\mathrm{p} / \mathrm{p}^{\circ}$, resultado un gráfico que mezcla las isotermas de los tipos II y III, característica de materiales macroporosos [24], lo cual explica su bajo valor de área superficial especifica. En la curva no se observa un loop de histéresis lo cual indica la ausencia o poca cantidad de mesoporos en el volumen de este material, lo cual también se evidencia por un promedio de poros de $28,2 \mathrm{~nm}$.

Tabla 3: Parámetros texturales de los sólidos calcinados a $500{ }^{\circ} \mathrm{C}$; Área superficial específica y diámetro de poros.

\begin{tabular}{ccc}
\hline MUESTRA & ÁREA SUPERFICIAL ESPECIFICA $\left(\mathbf{m}^{2} / \mathbf{g}\right)$ & DIÁMETRO DE POROS $(\mathbf{n m})$ \\
\hline $\mathrm{Zn}_{0,66} \mathrm{Al}_{0,33}$ & 10 & 28,2 \\
$\mathrm{Ni}_{0,66} \mathrm{Al}_{0,33}$ & 71 & 10,4 \\
$\mathrm{Zn}_{0,44} \mathrm{Ni}_{0,22} \mathrm{Al}_{0,33}$ & 72 & 31,2 \\
$\mathrm{Zn}_{0,33} \mathrm{Ni}_{0,33} \mathrm{Al}_{0,33}$ & 182 & 5,7 \\
$\mathrm{Ni}_{0,44} \mathrm{Zn}_{0,22} \mathrm{Al}_{0,33}$ & 177 & 6,4 \\
\hline
\end{tabular}

El tipo de isotermas originado por la muestra $\mathrm{Ni}_{0,66} \mathrm{Al}_{0,33}$ también es característica de un material predominantemente macroporoso, sin embargo, en esta se observa el punto de inflexión ausente en el sólido $\mathrm{Zn}_{0,66} \mathrm{Al}_{0,33}$. Este material a base de níquel y aluminio contiene poros con un radio de $10,4 \mathrm{~nm}$, lo que explica su mayor área superficial específica.

Los materiales conteniendo zinc, níquel y aluminio presentaron isotermas con loops de histéresis de diferente forma como se observa en la Figura 5. El sólido $\mathrm{Zn}_{0,44} \mathrm{Ni}_{0,22} \mathrm{Al}_{0,33}$ origino gráficos de adsorcióndesorción del tipo H3, característicos de materiales con mesoporos en la forma de hendiduras [24]. Este solido presento un área de $72 \mathrm{~m}^{2} / \mathrm{g}$ y diámetro de poros de $31,2 \mathrm{~nm}$, lo cual sugiere que su valor relativamente alto de área superficial especifica se debe a un mayor número de poros al comparase con sólidos como el $\mathrm{Ni}_{0,66} \mathrm{Al}_{0,33}$. 

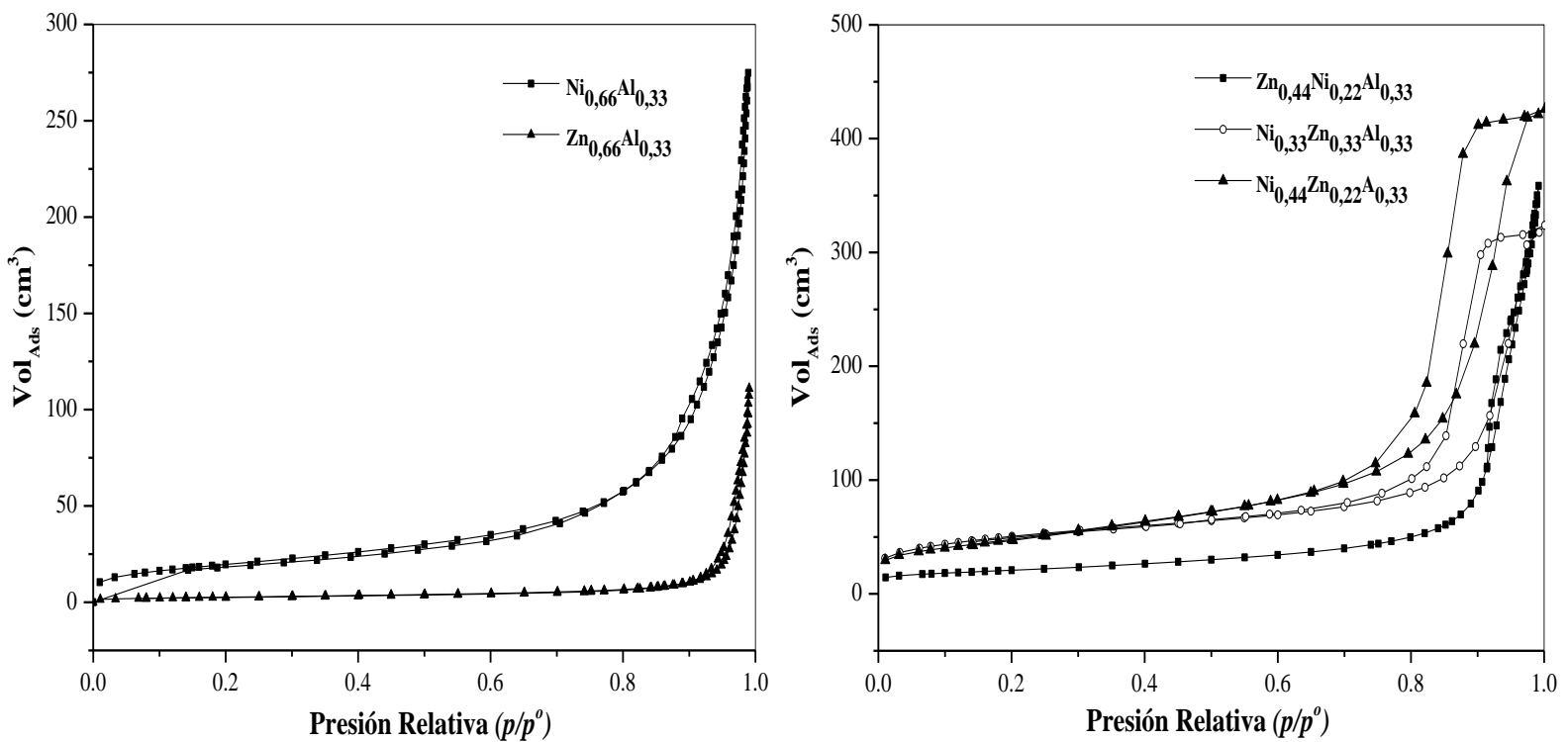

Figura 6: Isotermas de adsorción-desorción de nitrógeno a $77 \mathrm{~K}$ de los sólidos calcinados a $500{ }^{\circ} \mathrm{C}$.

Los catalizadores $\mathrm{Zn}_{0,33} \mathrm{Ni}_{0,33} \mathrm{Al}_{0,33}$ y $\mathrm{Ni}_{0,44} \mathrm{Zn}_{0,22} \mathrm{Al}_{0,33}$ presentaron isotermas del tipo $\mathrm{H} 2$ propias de materiales mesoporosos con poros estrechos en forma de capilares, formados por la aglomeración de partículas esféricas en el volumen de los materiales [24]. Estos materiales presentaron los mayores valores de área superficial específica, lo cual se debe principalmente a la presencia de poros con un menor radio de 5,7 y 6,4 $\mathrm{nm}$, tornando a estos solidos los más promisorios para actuar como catalizadores a base de zinc o níquel en reacciones en las que el área superficial especifica es de vital importancia.

\section{CONCLUSIONES}

La estructura del tipo hidrotalcita fue observada en todos los materiales, notándose que el aumento del contenido de zinc conduce a mayores valores del parámetro $a$ de las celdas ortorrómbicas de las hidrotalcitas. La disminución del parámetro $c$, condujo a una mayor interacción $\mathrm{CO}_{3}{ }^{2-} \cdot \cdots \mathrm{OH}$ en la estructura de las hidrotalcitas. Los sólidos con mayor contenido de zinc colapsaron a menores temperaturas, mientras que el material $\mathrm{Ni}_{0,66} \mathrm{Al}_{0,33}$ colapso a mayor temperatura. Los materiales calcinados contienen $\mathrm{ZnO}, \mathrm{NiO}$ y $\gamma-\mathrm{Al}_{2} \mathrm{O}_{3}$, así como la presencia de partículas grandes y con alta cristalinidad de la fase $\mathrm{ZnO}$, y de partículas pequeñas de $\mathrm{NiO}$, cuya cristalinidad está afectada por la presencia de cationes $\mathrm{Zn}^{2+}$ y $\mathrm{Al}^{3+}$ de este oxido. Los materiales conteniendo dos metales son materiales predominantemente macroporosos, sin embargo, el sólido a base de níquel-aluminio contiene también mesoporos que contribuyen con el aumento del área con respecto al material a base de zinc-aluminio. En relación a los materiales con trimetálicos, las estructuras son mesoporosas y con altas áreas superficiales específicas, observándose poros en forma de hendidura en el solido $\mathrm{Zn}_{0,44} \mathrm{Ni}_{0,22} \mathrm{Al}_{0,33}$ y poros estrechos en forma de capilares en los materiales $\mathrm{Zn}_{0,33} \mathrm{Ni}_{0,33} \mathrm{Al}_{0,33}$ y $\mathrm{Ni}_{0,44} \mathrm{Zn}_{0,22} \mathrm{Al}_{0,33}$.

\section{AGRADECIMIENTOS}

Los autores agradecen a la Universidad de Cartagena y al Servicio Nacional de Aprendizaje-SENA.

\section{BIBLIOGRAFÍA}

[1] CAVANI, F., TRIFIRO, F., VACCARI, A., "Hydrotalcite-type ânionic clays: Preparation, properties and application", Catalysis Today, v. 11, pp. 173-301, Dec. 1991.

[2] CREPALDI, E., BARROS, J., "Hidróxidos duplos lamelares: Síntese, estrutura, propriedades e aplicações”, Química Nova. v. 21, pp. 300-311, May. 1998.

[3] HE, J., WEI, M., LI, B., KANG, Y., EVANS, D., DUAN, X., "Preparation of layered double hydroxides”, In: Structure and Bonding, Springer-Verlag Berlin Heidelberg, pp. 89-119. 2005.

[4] NEWMAN, S., JONES, W., "Synthesis, characterization and applications of layered double hydroxides containing organic guests”, New Journal of Chemistry. v. 22, pp 105-115, Jan. 1998. 
[5] TORAISHI, T., NAGASAKI, S., TANAKA, S., "Adsorption behavior of $\mathrm{IO}_{3}{ }^{-}$by $\mathrm{CO}_{3}{ }^{2-}$ and $\mathrm{NO}_{3}{ }^{-}$hydrotalcite", Applied Clay Science. v. 22, pp. 17-23, Nov. 2002.

[6] FROST, R., MUSUMECI, A., MARTENS, W., ADEBAJO, M., BOUZAID, J., "Raman spectroscopy of hydrotalcites with sulphate, molybdate and chromate in the interlayer", Journal of Raman Spectroscopy. v. 36, pp. 925-931, Oct. 2005.

[7] DUONG, N., HANG, T., NICOLAY, A., PAINT, J., OLIVIER, M., "Corrosion protection of carbon steel by solvent free epoxy coatingcontaining hydrotalcites intercalated with different organic corrosion inhibitors", Progress in Organic Coatings, v. 101, pp. 331-341, Dec. 2016.

[8] EIBY, S., TOBLER, D., NEDEL, S., BISCHOFF, S., CHRISTIANSEN, B., HANSEN, A., KJAERGAARD, H., STIPP, S., "Competition between chloride and sulphate during the reformation of calcined hydrotalcite”, Applied Clay Science. v. 132-133, pp. 650-659, Nov. 2016.

[9] GOH, K., LIM, T., DONG, Z., "Application of layered double hydroxides for removal of oxyânions: A review", Water Research. V. 42, pp. 1343-1368, Nov. 2008.

[10] ZHANG, H., WANG, J., ZHANG, B., LIU, Q., LI, S., YAN, H., LIU, L., "Synthesis of a hydrotalcitelike compound from oil shale ash and its application in uranium removal. Colloids and Surfaces A: Physicochemical and Engineering Aspects. v. 444, pp. 129-137, Mar. 2014.

[11] QIANHE, L., CONGXIN, W., WEI, Q., BINGCHUN, W., ZHIJIAN, T., HUAIJUN, M., RENSHUN, $\mathrm{X}$., "The application of $\mathrm{Zr}$ incorporated $\mathrm{Zn}-\mathrm{Al}$ dehydrated hydrotalcites assolid base in transesterification", Catalysis Today. v. 234, pp.1-6. Oct. 2014.

[12] MEZA, E., FARO, A., DE FREITAS, T., ASSAF, J., RANGEL, M., “A comparison between copper and nickel-based catalysts obtained from hydrotalcite-like precursors for WGSR", Catalysis Today. v. 171, pp. 290-296, Aug. 2011.

[13] MEZA-FUENTES, E., CADETE-SANTOS, F., PRAKASH, S., DA COSTA-FARO, A., DE FREITASSILVA, T., MANSUR-ASSAF, J., RANGEL, M., "The effect of metal content on nickel-based catalysts obtained from hydrotalcites for WGSR in one step", International Journal of Hydrogen Energy, v. 39, pp.1-14, Jan. 2014.

[14] MESA, S., ARBOLEDA, J., AMAYA, S., ECHAVARRÍA, C., "Hidrotalcitas de modificadas con V y $\mathrm{Cr}$ como precursores de catalizadores para deshidrogenación oxidativa de propano", Ingenieria y Competitividad, v. 14, pp. 169-178, Nov. 2012.

[15] GUERRERO, P., GARCÍA, C., MORENO, R., MÉRIDA, J., SANTAMARÍA-GONZÁLEZ, J., JIMÉNEZ, A., MAIRELES, P., "Glycerol valorization by etherification to polyglycerols by using metal oxides derived from MgFe hydrotalcites", Applied Catalysis A: General, v. 470, pp. 199-207, Jan. 2014.

[16] CHEN, F., WANG, G., LI, W., YANG, F., "Glycolysis of Poly(ethylene terephthalate) over Mg-Al Mixed Oxides catalysts derived from hydrotalcites", Industrial \& Engineering Chemistry Research, v. 52, pp. 565-571, Dec. 2012.

[17] BRUNAUER, S., EMMETT, P., TELLER, E., "Adsorption of Gases in Multimolecular Layers", Journal of the American Chemical Society, v. 60, pp. 309-319, Feb. 1938.

[18] BARRETT, E., JOYNER, L., HALENDA, P., "The Determination of pore volume and area distributions in porous substances. I. computations from nitrogen isotherms", Journal of the American Chemical Society, v. 73, pp. 373-380, Jan. 1951.

[19] JITIANU, M., BÃLÃSOIU, M., MARCHIDAN, R., ZAHARESCU, M., CRISAN, D., CRAIU, M., "Thermal behaviour of hydrotalcite-like compounds: study of the resulting oxidic forms", International Journal of Inorganic Materials, v. 2, pp. 287-300, Jun. 2000.

[20] COSTANTINO, U., MARMOTTINI, F., SISANI, M., MONTANARI, T., RAMIS, G., BUSCA, G., TURCO, M., BAGNASCO, G., "Cu-Zn-Al hydrotalcites as precursors of catalysts for the production of hydrogen from methanol", Solid State Ionics, v. 176, pp. 2917-2922, Dec. 2005.

[21] KLOPROGGE, J., WHARTON, D., HICKEY, L., FROST, R., "Infrared and Raman study of interlayer ânions $\mathrm{CO}_{3}{ }^{2-}, \mathrm{NO}_{3}{ }^{-}, \mathrm{SO}_{4}{ }^{2-}$ and $\mathrm{ClO}_{4}{ }^{-}$in $\mathrm{Mg} / \mathrm{Al}$ hydrotalcite", American Mineralogist, v. 87, pp. 623-629, May. 2002.

[22] MELIÁN-CABRERA, I., LÓPEZ-GRANADOS, M., FIERRO, J., "Thermal decomposition of a hydrotalcite-containing $\mathrm{Cu}-\mathrm{Zn}-\mathrm{Al}$ precursor: Thermal methods combined with an in situ DRIFT study. Physical Chemistry Chemical Physics, V. 43, pp. 3122-3127, May. 2002. 
[23] RÍOS-LEÓN, I., SOLANO-POLO, C., RODRÍGUEZ-RUIZ, J., ESPINOSA-FUENTES, E., MEZAFUENTES, E., "Study by infrared spectroscopy and thermogravimetry of the effect of temperature on nickelaluminum hydrotalcites", Dyna, v. 84, pp. 9-16, Jun. 2017.

[24] SING, K., EVERETT, D., HAUL, R., MOSCOU, L., PIEROTTI, R., ROUQUEROL, J., SIEMIENEWSKA, T., "Reporting physisorption data for gas/solid systems, with special reference to the determination of surface area and porosity", Pure \& Applied. Chemistry, v. 57, pp. 603-619, Apr. 1985.

\section{ORCID}

Johana Rodríguez-Ruiz Shirley Osorio-Herrera

http://orcid.org/0000-0002-4263-4242

Edgardo Meza-Fuentes https://orcid.org/0000-0003-0485-8293

http://orcid.org/0000-0003-3725-8112 